\title{
Gaia 2.0
}

\section{Could humans add some level of self-awareness to Earth's self-regulation?}

\author{
$B y$ Timothy M. Lenton and Bruno Latour
}

According to Lovelock and Margulis' Gaia hypothesis, living things are part of a planetaryscale self-regulating system that has maintained habitable conditions for the past 3.5 billion years $(1,2)$. Gaia has operated without foresight or planning on the part of organisms, but the evolution of humans and their technology are changing that. Earth has now entered a new epoch termed the Anthropocene (3), and humans are beginning to become aware of the global consequences of their actions. As a result, deliberate self-regulation-from personal action to global geoengineering schemes-is either happening or imminently possible. Making such conscious choices to operate within Gaia constitutes a fundamental new state of Gaia, which we term Gaia 2.0. By emphasizing the agency of lifeforms and their ability to set goals, Gaia 2.0 may be an effective framework for fostering global sustainability.

At first sight, the potential for a successful Gaia 2.0 does not seem promising. First, despite large-scale mobilization of scientists, activists, and citizens, large parts of the human population are indifferent to the Anthropocene, and many deny anthropogenic climate change (4). Second, there is no proof that consciousness in this context is anything but the belated and retrospective realization that mistakes had been made and might be partially redressed. Indeed, the first formulation of the Gaia hypothesis (1) is almost exactly contemporary with what is now seen as the start of the Anthropocene (3). Third, the examples of social Darwinism, sociobiology, and dialectical materialism suggest that drawing political lessons from nature is problematic.

Nevertheless, it is important to have a second look at the connection between the original Gaia concept and a possible Gaia 2.0, because the original Gaia has many traits that were not detectable in earlier notions of nature associated with the development of Western civilization. Before the Anthropocene, Western societies saw themselves as the only conscious agents in a passive material environment. Today, they must cope with the brutal reactions of living organisms that are continually reshaping their surroundings, creating in part their own conditions for survival $(4,5)$. Gaia thus establishes a new continuity between humans and non- humans that was not visible before-a relation between free agents (4). This understanding offers the potential to learn from features of Gaia to create a Gaia 2.0. We focus here on three of these features: autotrophy, network, and heterarchy.

\section{Autotrophy}

Autotrophs use free energy to continually (re)make themselves out of simple substances that are present in their surroundings. The Earth surface where most of the biosphere resides is a very nearly materially closed system. Hence, like an autotroph, the collective flourishing of life for the past 3.5 billion years has depended on the internal recycling of materials, powered by solar energy (6). The origin of these material recycling loops is at least partially understood (7). There needs to be a source of free energy to support recycling, which usually comes from the Sun and enters the system via photosynthetic primary producers. Recycling is built on metabolic byproducts, where one organism's waste becomes another's food. Closure of a recycling loop triggers a self-perpetuating feedback process: The participants in the recycling loop are no longer limited by what comes into their world, but rather by how efficiently they can recycle resources. For example, coral reefs and the Amazon rainforest thrive on recycling in otherwise low-nutrient conditions.

If, by contrast, we consider the state of the technosphere in the Anthropocene (5), an audit made by Gaia would question the purported quality of many innovations and note that from an engineering point of view, they perform poorly. Humans currently extract fossil energy, rock phosphate, and other raw materials from the crust far faster than they would normally come to the surface, and then dump the waste products on land, in the atmosphere, and in the ocean. Compared to Gaia, this is a very badly coupled and unsustainable set of inventions.

This does not mean that humans should stop inventing, but rather that engineering should shift attention to become as smart as Gaia in achieving nearly closed material cycling powered by sustainable energy. The input of solar energy has the potential to far outstrip current fossil energy consumption, and renewables are rapidly becoming cost- competitive with fossil fuel energy for electricity generation (8). There should thus be no long-term shortage of energy. The challenge is to design and incentivize a transition to a circular economy. As in the original Gaia this must be built on waste products becoming useful resources to make new products. Despite practical obstacles and thermodynamic constraints, there is huge potential to increase material recycling in Gaia 2.0 (9).

\section{Networks}

Gaia was built by adaptive networks of microbial actors that exchanged materials, electrons, and information (10), the latter though ubiquitous horizontal gene transfer. These microbial networks form the basis of the recycling loops that make up global biogeochemical cycles. Functional roles in these networks have been retained even whilst the taxa performing them were replaced (11). Therefore, sufficient biodiversity to provide functional redundancy contributes to the robust self-regulation of Gaia.

Microbial networks also created longlived products that sometimes accumulated globally - notably oxygen in the atmosphere. This in turn facilitated an increase in the diversity of life and metabolisms, and enabled the evolution of new levels of biological organization and connectedness (6), with new mechanisms of coordination. Humans and our adaptive social networks are the latest realization of this.

In Gaia 2.0, horizontal transfer of information, functional diversity with redundancy, and distributed control will likely be important to a successful circular economy. The challenge is to support diverse, auto-catalytic networks of human agents that can propel transformations toward goals such as sustainable energy, fueling the efficient cycling of resources. This is particularly challenging given a social and economic paradigm of short-term localized gain and relatively weak global, unifying, long-term structures to counteract this.

\section{Heterarchy}

Depending on the scale and time span considered, completely different mechanisms are at work within Gaia (7). Such heterarchy is especially visible in the climate regulation that has taken so much political importance 
of late. Some of Earth's climate self-regulation mechanisms (6) are purely physical and chemical, but many involve biology. On time scales of hundreds of thousands of years, changes in global temperature are counteracted by biologically amplified changes in the removal of $\mathrm{CO} 2$ by silicate weathering. On intermediate time scales of millennia, the dissolution of carbonate sediments on land and the ocean floor increases $\mathrm{CO} 2$ storage in the ocean. On even shorter time scales of years to centuries, land and ocean carbon sinks roughly halve the rate of $\mathrm{CO}_{2}$ rise and climate change.

Thus, each mechanism in Gaia has its own capacity for resistance and expansion. Natural selection can only help to explain environmental regulation at small scales of space and time (7). At large space and time scales, simpler dynamical mechanisms are at play (7): Systems that find self-stabilizing configurations tend to persist (12), and systems that persist have a greater likelihood of acquiring further persistence-enhancing properties $(11,13)$. Through these cruder selection mechanisms, Earth may have acquired and accumulated stabilizing feedback mechanisms involving life (7).

The upshot is that Gaia's self-regulation of climate is probably fairly crude compared to its efficient recycling of resources. The recent glacial-interglacial cycles indicate that the climate system can be quite unstable and thus vulnerable to human interference, which has already increased atmospheric $\mathrm{CO}_{2}$ to levels last seen 3 to 5 million years ago. This heterarchy of mechanisms of different reliability makes the task of Gaia 2.0 to restabilize the climate especially daunting. Simultaneously, humans are altering nutrient cycles relatively more than the carbon cycle, posing an additional challenge for Gaia 2.0 to restabilize nutrient cycling.

Implementation of alternative forms of climate control in order to reduce production of $\mathrm{CO}_{2}$ or augment existing feedbacks (14) depends on who is in charge of such voluntary activity. The results would clearly be different if the Intergovernmental Panel on Climate Change, President Putin, the Californian legislature, or President Trump has the finger on the proverbial thermostat. In reality, all these agents and many others have some grip on the thermostat, and their combined effect is not simple to predict.

\section{Politics}

Drawing a parallel between the original Gaia concept and a possible Gaia 2.0 gives an occasion to re-evaluate our collective goals as well as the means of achieving them. A central goal for this century is surely to achieve a flourishing future for all life on this planet, including a projected 9 to 11 billion people. Human flourishing is not possible without a biodiverse, life-sustaining Earth system. This is recognized in the United Nations' 17 Sustainable Development Goals. But achieving those goals requires that human societies exercise self-aware self-regulation (14).

Yet, maintaining a self-regulating, human life-supporting planet is not the primary goal of some dominant modes of collective human activity today. Despite a flood of monitoring information, present industrial societies seem less able to track change in their environment than the life forms that compose Gaia, because that information is often ignored where it matters by those in power. It's as if purposelessness had shifted from the natural to the social domain.

There is clearly at this point a political question of orientation toward or away from the lessons to be drawn from Gaia. This creates a conflict that takes precedence over all others. The climate science controversies demonstrate that scientists are now drawn into knowledge and power struggles for which they have not been prepared. This does not mean that people inspired by Gaia will be endowed with deeper foresight. In matters of politics, it is prudent to follow John Dewey's advice (15) that we cannot expect to know the best solution in advance, but only that we can improve the quality of the sensors that detect shortcomings and the speed with which we rectify the course. If in politics the blind lead the blind, then hope rests on finding the best way to activate the white cane to fumble in the dark.

This is where the scientific establishment becomes crucial in multiplying the sensors, improving their qualities, speeding the dissemination of their results, accelerating the quality of the models making sense of those data, and proposing alternative explanations to phenomena. Such an infrastructure is not, however, limited to scientists: They have to collaborate with citizens, activists, and politicians to quickly realize where things are going wrong.

Creating an infrastructure of sensors that allows tracking the lag time between environmental changes and reactions of societies is the only practical way in which we can hope to add some self-awareness to Gaia's self-regulation. This framing of the problem gives a clear ethical direction: Any attempt to tamper with the sensors or slow down the reaction to errors jeopardizes the chance to learn from Gaia how to close the loops that would make Gaia 2.0 a better world to sustain the human population than the present one.

REFERENCES AND NOTES:

1. J. E. Lovelock, Atmos. Environ. 6, 579 (1972).

2. J. E. Lovelock, L. M. Margulis, Tellus 26, 2 (1974).

3. C. N. Waters et al., Science 351, aad2622 (2016).

4. B. Latour, Facing Gaia: Eight Lectures on the New Climatic Regime (Polity, 2017).

5. P. K. Haff, Technology as a geological phenomenon: implications for human well-being, Geological Society, London, Special Publications 395, 301 (2013).

6. T. M. Lenton, A. J. Watson, Revolutions that made the Earth. (0xford Univ. Press, Oxford, 2011)

7. T. M. Lenton et al., Tr. Ecol. Evol. 33, 633 (2018).

8. IRENA, Renewable Power Generation Costs in 2017 (International Renewable Energy Agency, Abu Dhabi, 2018).

9. L. Ciacci, E. M. Harper, N. T. Nassar, B. K. Reck, T. E. Graedel, Environ Sci. Technol. 50, 11394 (2016)

10. P. G. Falkowski, T. Fenchel, E. F. Delong, Science 320, 1034 (2008).

11. W. F. Doolittle. J. Theor. Bio. 434, 11 (2017)

12. A. Wagner, Robustness and Evolvability in Living Systems. S. A. Levin, S. H. Strogatz, Eds., Princeton Studies in Complexity (Princeton University Press, 2007)

13. W. F. Doolittle, Biol. Philos. 29, 415 (2014)

14. O. Morton, The Planet Remade: How Geoengineering Could Change the World (Princeton Univ. Press, Princeton, NJ, 2015).

15. J. Dewey, The Public and its Problems (Henry Holt and Company, 1927).

Acknowledgments: We thank the Giorgio Cini Foundation for bringing us together, two reviewers, Sebastien Dutreuil, and the participants in the dialogue 'What's the Body of the Body Politic? Sovereignty, Identity, Ecology' for valuable feedback, and the Zentrum fur Media Kuntz of Karlsruhe for support. 